\title{
The roles of ING5 in gliomas: a good marker for tumorigenesis and a potential target for gene therapy
}

\author{
Shuang Zhao ${ }^{1}$, Zhi-Juan Zhao ${ }^{2}$, Hao-Yu He ${ }^{1}$, Ji-Cheng Wu ${ }^{1}$, Xiao-Qing Ding ${ }^{1}$, Lei \\ Yang $^{1}$, Ning Jia ${ }^{2}$, Zhi-Jie Li ${ }^{1}$ and Hua-Chuan Zheng ${ }^{1}$ \\ ${ }^{1}$ Department of Experimental Oncology and Animal Center, Shengjing Hospital of China Medical University, Shenyang \\ 110004, China \\ ${ }^{2}$ The First Affiliated Hospital of Jinzhou Medical University, Jinzhou 121001, China
}

Correspondence to: Hua-Chuan Zheng, email: zheng_huachuan@hotmail.com

Keywords: glioma, ING5, tumorigenesis, chemotherapy, gene therapy

Received: December 16, $2016 \quad$ Accepted: April 28, $2017 \quad$ Published: May 11, 2017

Copyright: Zhao et al. This is an open-access article distributed under the terms of the Creative Commons Attribution License 3.0 (CC BY 3.0), which permits unrestricted use, distribution, and reproduction in any medium, provided the original author and source are credited.

\section{ABSTRACT}

To elucidate the anti-tumor effects and molecular mechanisms of ING5 on glioma cells, we overexpressed it in $U 87$ cells, and examined the phenotypes and their relevant molecules. It was found that ING5 overexpression suppressed proliferation, energy metabolism, migration, invasion, and induced $G_{2} / M$ arrest, apoptosis, dedifferentiation, senescence, mesenchymal- epithelial transition and chemoresistance to cisplatin, MG132, paclitaxel and SAHA in U87 cells. There appeared a lower expression of $\mathbf{N}-$ cadherin, Twist, Slug, Zeb1, Zeb2, Snail, Ac-H3, Ac-H4, Cdc2, Cdk4 and XIAP, but a higher expression of Claudin 1, Histones 3 and 4, p21, p53, Bax, $\beta$-catenin, PI $\mathrm{K}$, Akt, and p-Akt in ING5 transfectants. ING5 overexpression suppressed tumor growth of $U 87$ cells in nude mice by inhibiting proliferation and inducing apoptosis. Downregulated ING5 expression was closely linked to the tumorigenesis and histogenesis of glioma. These data indicated that ING5 expression might be considered as a good marker for the tumorigenesis and histogenesis of gliomas. It might be employed as a potential target for gene therapy of glioma. $\mathrm{PI}_{3} \mathrm{~K} / \mathrm{Akt}$ or $\beta$-catenin/TCF-4 activation might be positively linked to chemotherapeutic resistance, mediated by ING5.

\section{INTRODUCTION}

Glioma is a most commonly occurring highly malignant primary brain tumor, but its molecular pathways of pathogenesis remain unclear [1]. Although major advancements have been made in surgery, chemoradiotherapy, gene therapy, and immunotherapy of glioma, a poor prognosis remains characteristic of the tumor [2]. The active migration of the glioma cells through the narrow extracellular spaces in the brain makes the glioma cells elusive targets for effective surgical treatment [3].

Since the discovery of class II tumor suppressor ING1 in 1996, five different ING genes (ING1 to ING5) encode the proteins with highly-conserved plant homeodomain motifs, which induces apoptosis, cell arrest, senescence and DNA repair as an important cofactor of p53 [4-6]. Further, they have emerged as a versatile family of growth regulators, phospholipid effectors, histone mark sensors, core components of HDAC1/2 and HAT chromatin-modifying complexes. It has been reported that ING5 inhibit proliferation, cell cycle progression, migration and invasion and epithelialmesenchymal transition (EMT) of tumor cells [7-9]. ING5 overexprssion was demonstrated to promote glucose catabolism and fat accumulation in lung cancer cells by up-regulating the expression of ADFP, PFK-1, PDPc and HXK1 [10]. Reportedly, ING5 expression was decreased in many malignancies, including breast cancer [11], bladder cancer [12], gastric cancer [13], lung cancer [14], head and neck squamous cell carcinoma [15]. Recently, miR-331-3p and miR-193a-3p might suppress ING5 expression to increase cell proliferation and decrease multi- chemoresistance respectively $[12,16]$. Liu et al. [17] found that down-regulated ING5 expression was detected in cells transfected with miR-196a precursor, 
and accompanied by less apoptosis, higher invasion and proliferation of pancreatic cancer cells.

Here, we observed the effects of ING5 overexpression on the anti-tumor and relevant molecular mechanisms of glioma cells. ING5 overexpression was found to suppress proliferation, energy metabolism, migration, invasion, and induced $\mathrm{G}_{2}$ arrest, apoptosis, dedifferentiation, senescence, MET, and chemotherapeutic resistance of U87 cells. We also observed the anti-tumor effects of ING5 in xenograft models of nude mice and clarified the molecular mechanisms. A lower expression of cytoplasmic and nuclear ING5 was for the first time observed in gliomas than normal brain tissues.

\section{RESULTS}

\section{The effects of ING5 overexpression on biological phenotypes of glioma cells}

After transfected with pEGFP-N1-ING5, U87 cell showed ING5 overexpression at both mRNA and protein levels ( $p<0.05$, Figure $1 \mathrm{~A}$ and $1 \mathrm{~B})$. ING5 overexpression reduced proliferation, glycolytic function and mitochondrial respiration, and alkaline phosphatase (ALP) activity of glioma cells $(p<0.05$, Figure $1 \mathrm{C}-1 \mathrm{E})$. PI staining showed that ING5 overexpression induced $\mathrm{G}_{2} / \mathrm{M}$ arrest and apoptosis in U87 cells ( $p<0.05$, Figure $1 \mathrm{~F}$ and $1 \mathrm{G})$. The wound healing and transwell assays demonstrated that ING5 decreased cell migration and invasion ( $p<0.05$, Figure $1 \mathrm{H}$ and $1 \mathrm{I})$. ING5 also promoted the senescence of U87 cells, evidenced by $\beta$-galactosidase staining (Figure 1J). After the exposure to cisplatin, MG132, paclitaxel, and SAHA, U87 transfectants showed higher viability and lower apoptosis than the control in both dose- and time-dependent manners $(p<0.05$, Figure 2A and 2B).

\section{The effects of ING5 overexpression on the expression and transcriptional activity of phenotype-related proteins in glioma cells}

As shown in Figure 3A, the protein expression levels of Claudin 1, Histones 3 and 4, p21, p53, Bax, $\beta$-catenin, $\mathrm{PI}_{3} \mathrm{~K}$, Akt and p-Akt in ING5 transfectants were higher than those observed in the control and mock cells, while versa for N-cadherin, Twist, Slug, Zeb1, Zeb2, Snail, Ac-H3, Ac-H4, Cdc2, Cdk4 and XIAP. Dual luciferase reporter gene assay demonstrated that both TCF-4 promoter activity and TCF4- mediated gene transcriptional activity became higher in ING5 transfectants than the control and mock cells ( $p<0.05$, Figure 3B).

\section{ING5 suppressed the tumor growth of glioma cells in xenograft model}

As shown in Figure 4A-4C, the tumor volume and weight of ING5 transfectant xenografts become smaller than the control by calculation and weighting $(p<0.05)$. The inhibitory effect was positively correlated with a low proliferation and a high apoptosis in comparison to the control, evidenced by ki-67 immunostaining and TUNEL assay (Figure 4D).

\section{Relationship between ING5 expression and clinicopathological features of glioma}

ING5 expression was lower in gliomas than that in normal brain tissue regardless of its subcellular expression pattern ( $p<0.05$, Figure 5A, Table 1). A higher expression of cytoplasmic or nuclear ING5 was detected in anaplastic astrocytoma than astrocytoma and glioblastoma ( $p<0.05$, Table 2). Then, we used Lee's, Bredel's, Sun's and Murat's dataset to perform bioinformatics analysis and found that ING5 mRNA expression was higher in glioblastoma than normal brain tissue from the results of the latter two investigators $(p<0.05$, Figure $5 \mathrm{~B})$. There were no other data for the other subtypes of gliomas.

\section{DISCUSSION}

ING5 is localized to human chromosome $2 \mathrm{q} 37.3$, contains 8 exons and 7 introns, and encodes 5233 bp cDNA and $28 \mathrm{kDa}$ protein of 240 amino acids. ING5 belongs to candidate tumor suppressor, interacts with histone $\mathrm{H} 3 \mathrm{~K} 4 \mathrm{me} 3$, and promotes the formation of two different HAT complexes to control the histone acetylation [5]. In the present study, lower cytoplasmic and nuclear expression of ING5 in a large number of glioma cases suggested that its down-regulation promoted the tumorigenesis of glioma. ING5 overexpression in anaplastic astrocytoma indicated a close link between ING5 protein and the tumorigenesis of anaplastic atrocytoma. Recently, a low expression of ING5 mRNA was detectable in oral squamous cell carcinoma [9], but versa from Sun and Burat. The hypoexpression of nuclear ING5 protein and its nucleocytoplasmic translocation were observed in colorectal and gastric cancers, and closely correlated with their aggressive behaviors or adverse prognosis [18, 19].

Reportedly, ING5 overexpression diminished colony-forming efficiency, cell population in $\mathrm{S}$ phase, and caused an induction of apoptosis in a p53-dependent manner [20]. In line with the other reports [10, 11, 13, 14], we found that ING5 overexpression inhibited proliferation, glycometabolism, invasion and migration, induced apoptosis, cell cycle arrest, senescence and differentiation of glioma cells. Additionally, ING5 also suppressed the tumor growth of glioma by inhibiting proliferation and inducing apoptosis in tumor-bearing nude mice, in agreement with our in vitro data $[10,13]$. In combination of these findings, we hypothesized that ING5 might reverse the aggressive phenotypes of glioma cells and be employed as a potential target for gene therapy of glioma. 


\section{A}

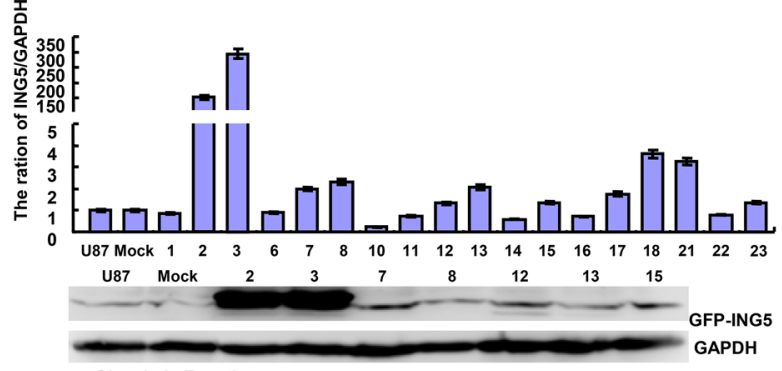

D
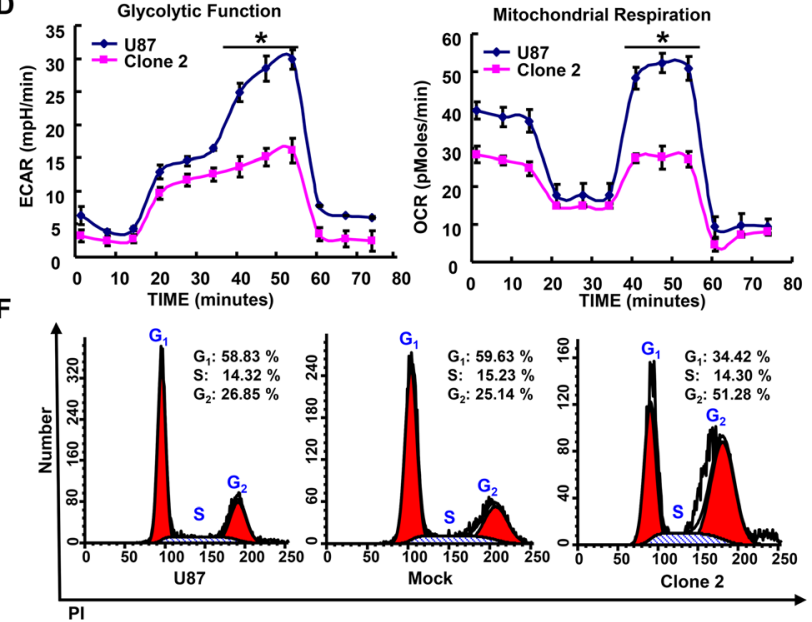

G
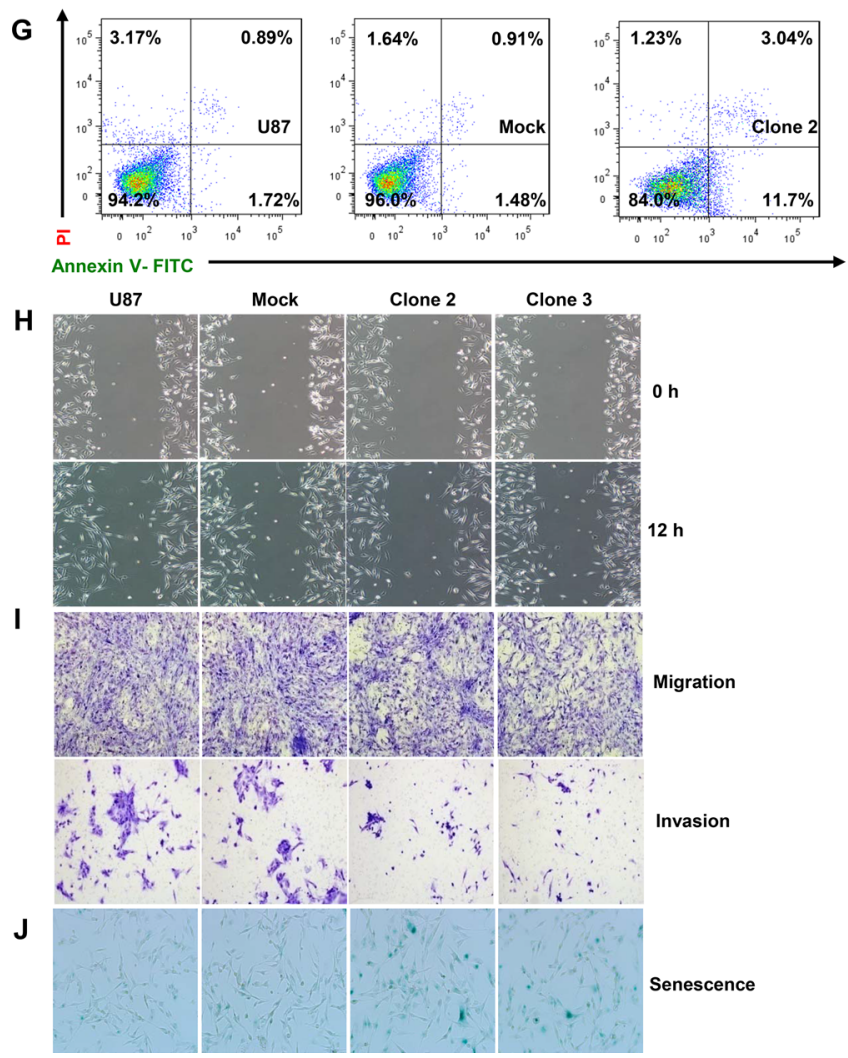

C

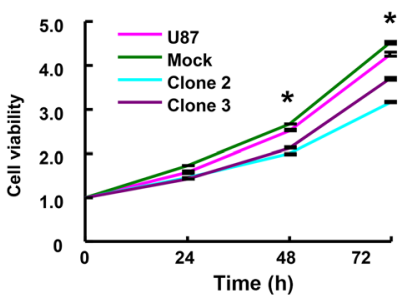

E

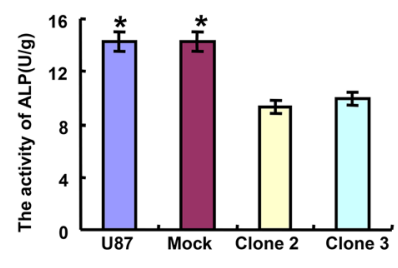

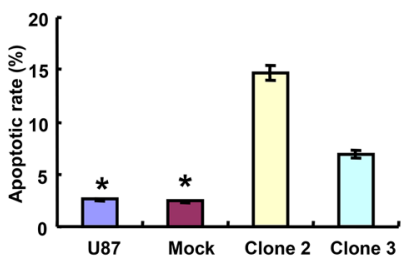
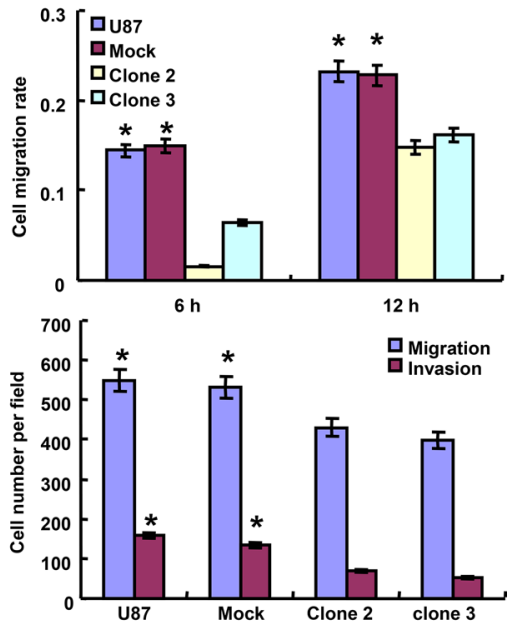

Figure 1: Effect of ING5 on aggressive phenotypes of glioma cells. After transfection of pEGFP-N1-ING5, ING5-overexpressing clones were selected by realtime PCR (A) and western blot (B). ING5 overexpression suppressed proliferation by MTT assay (C), glucolysis and mitochondrial respiration by cellular energy metabolism assay (D), differentiation by ALP activity (E), and induced $\mathrm{G}_{2} / \mathrm{M}$ arrest by PI staining $(\mathbf{F})$, apoptosis by Annexin-V staining $(\mathbf{G})$ in U87 cells. ING5 decreased the ability of U87 cells to migrate and invade, evidenced by wound healing $(\mathbf{H})$ and transwell (I) assays. ING5 overexpression also promoted the $\mathrm{U} 87$ cell senescence by $\beta$-galactosidase staining $(\mathbf{J})$, $* p<0.05$. 
Table 1: ING5 expression in glioma tissues

\begin{tabular}{|c|c|c|c|c|c|c|c|c|c|c|c|c|c|}
\hline \multirow{2}{*}{ Groups } & \multirow{2}{*}{$n$} & \multicolumn{6}{|c|}{ Nuclear ING5 expression } & \multicolumn{6}{|c|}{ Cytoplasmic ING5 expression } \\
\hline & & - & + & ++ & +++ & PR(\%) & $p$ & - & + & ++ & +++ & PR(\%) & $p$ \\
\hline Normal tissue & 56 & 24 & 20 & 7 & 5 & 57.1 & 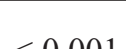 & 14 & 19 & 19 & 4 & 75.0 & \\
\hline Glioma & 312 & 190 & 32 & 43 & 47 & 39.1 & 0.001 & 152 & 73 & 67 & 20 & 51.3 & 0.001 \\
\hline
\end{tabular}

$\mathrm{PR}=$ positive rate.

Li et al. [12] reported that ING5 knockdown increased the chemoresistance and inhibited the DNA damage response pathway in 5637 cells. Mendes-Pereira et al. [21] found that ING5 silencing causes sensitivity to this endocrine agent, tamoxifen in breast cancer cells. Consistent with the results in gastric and lung cancer cells $[10,13]$, ING5 mediated chemoresistance of glioma cells to various anti-tumor drugs, which paralleled with apoptotic resistance. $\mathrm{PI}_{3} \mathrm{~K} / \mathrm{Akt} / \mathrm{p} 70 \mathrm{~S} 6 \mathrm{~K}$ pathway is reported to enhance cellular survival and suppress apoptosis because Akt1 inactivates Caspase 9 by phosphorylation on ser196, and disassociates BAD from Bcl-2 or Bcl-xL to lose its pro-apoptotic effect $[22,23]$. Wnt signaling pathway can inhibit GSK-3mediated phosphorylation of $\beta$-catenin for the entry of the latter into the nucleus, where the interaction of $\beta$-catenin with TCF family transcription factors increases the transcription c-myc, VEGF, survivin and Cyclin DI [24]. Here, $\mathrm{PI}_{3} \mathrm{~K}$, Akt, p-Akt and $\beta$-catenin were overexpressed,
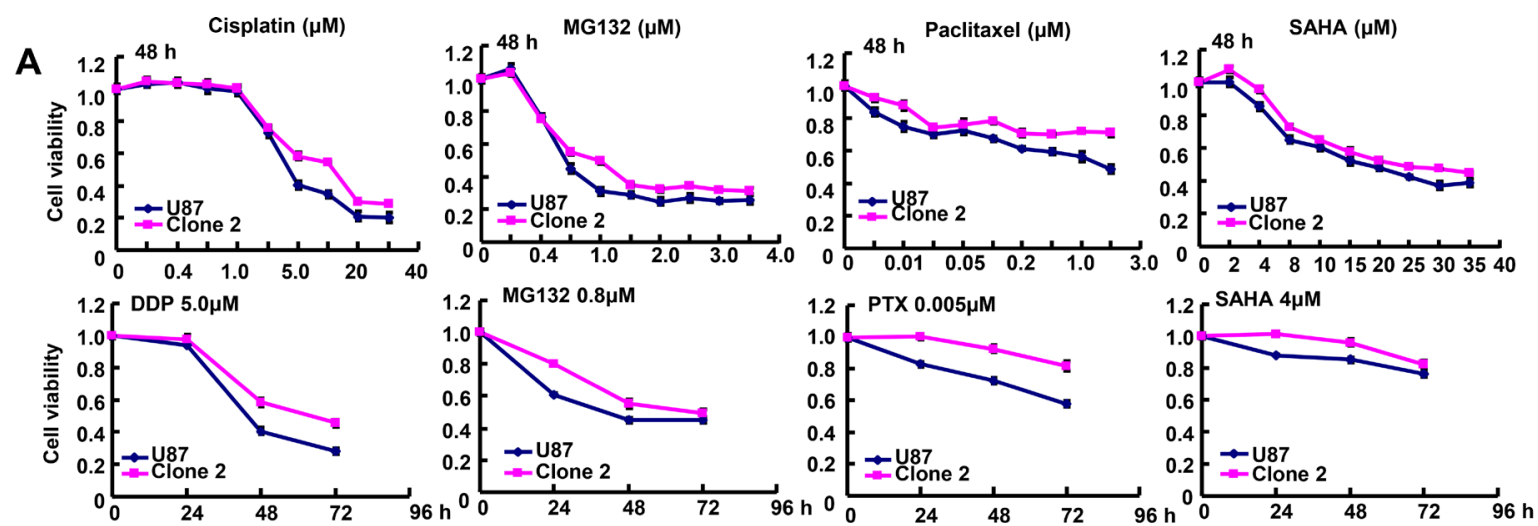

B
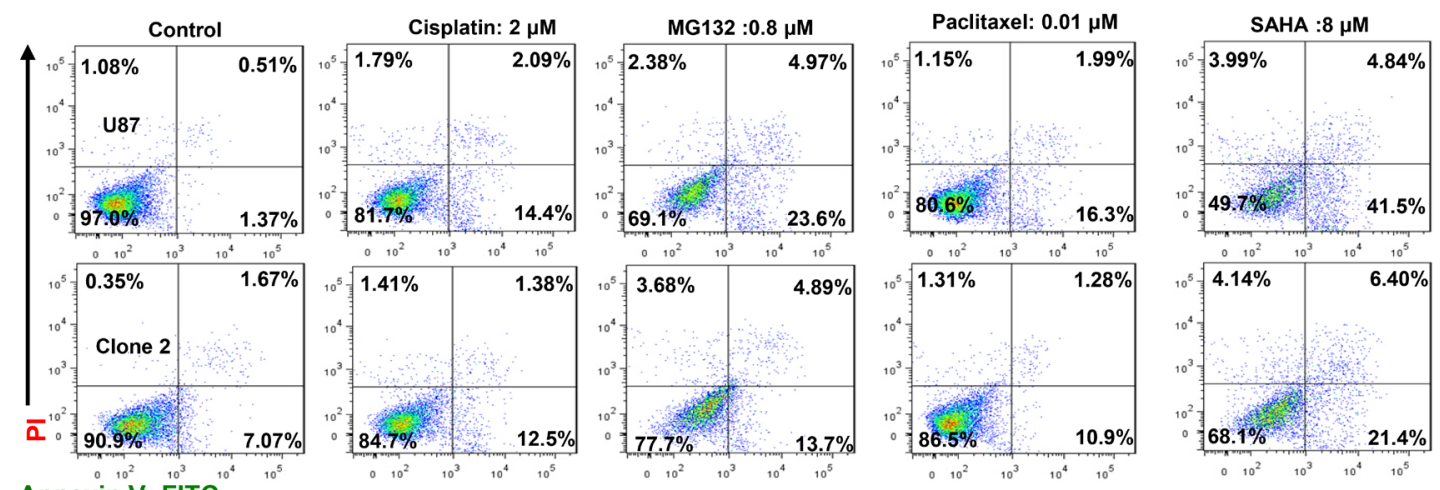

C
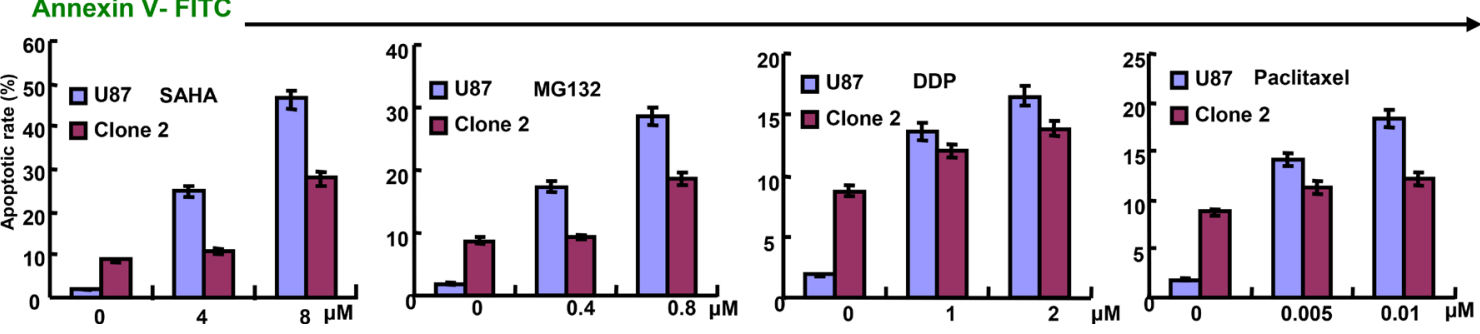

Figure 2: ING5 attenuated the sensitivity of U87 to chemotherapeutic agents. After exposed to cisplatin (DDP), MG132, paclitaxel, and SAHA, ING5 transfectants showed a higher proliferation (A) and a lower apoptotic level (B) than the control in both concentration- and time-dependent manners. 
Table 2: Relationship between ING5 expression and the clinicopathological features of gliomas

\begin{tabular}{|c|c|c|c|c|c|c|c|c|c|c|c|c|c|}
\hline \multirow{2}{*}{$\begin{array}{l}\text { Clinicopathological } \\
\text { features }\end{array}$} & \multirow{2}{*}{$n$} & \multicolumn{6}{|c|}{ Nuclear ING5 expression } & \multicolumn{6}{|c|}{ Cytoplasmic ING5 expression } \\
\hline & & - & + & ++ & +++ & PR(\%) & $p$ & - & + & ++ & +++ & $\operatorname{PR}(\%)$ & $p$ \\
\hline \multicolumn{14}{|l|}{ Sex } \\
\hline Male & 204 & 113 & 16 & 28 & 47 & 44.6 & \multirow{2}{*}{0.601} & 100 & 52 & 38 & 14 & 51.0 & \multirow{2}{*}{0.731} \\
\hline Female & 156 & 86 & 18 & 26 & 26 & 44.9 & & 77 & 31 & 38 & 10 & 50.6 & \\
\hline \multicolumn{14}{|l|}{ Age/years } \\
\hline$<44$ & 161 & 91 & 12 & 20 & 38 & 43.5 & \multirow{2}{*}{0.843} & 78 & 42 & 33 & 8 & 51.6 & \multirow{2}{*}{0.803} \\
\hline$\geq 44$ & 198 & 108 & 21 & 34 & 35 & 45.5 & & 99 & 41 & 42 & 16 & 50.0 & \\
\hline \multicolumn{14}{|l|}{ Grading } \\
\hline I-II & 150 & 93 & 11 & 16 & 30 & 38.0 & \multirow{2}{*}{0.876} & 72 & 41 & 32 & 5 & 52.0 & \multirow{2}{*}{0.625} \\
\hline III-IV & 118 & 70 & 14 & 20 & 14 & 40.7 & & 61 & 30 & 21 & 6 & 48.3 & \\
\hline $\begin{array}{l}\text { Histological } \\
\text { classification }\end{array}$ & & & & & & & - & & & & & & - \\
\hline Astrocytoma & 176 & 124 & 10 & 13 & 29 & 29.5 & & 86 & 50 & 29 & 11 & 51.1 & \\
\hline $\begin{array}{l}\text { Anaplastic } \\
\text { astrocytoma }\end{array}$ & 42 & 14 & 6 & 11 & 11 & $66.7^{*}$ & & 15 & 7 & 14 & 6 & $64.3^{*}$ & \\
\hline Glioblastoma & 60 & 33 & 13 & 11 & 3 & 45.0 & & 35 & 12 & 11 & 2 & 41.7 & \\
\hline
\end{tabular}

$* p<0.05$, compared with astrocytoma and gliblastoma.
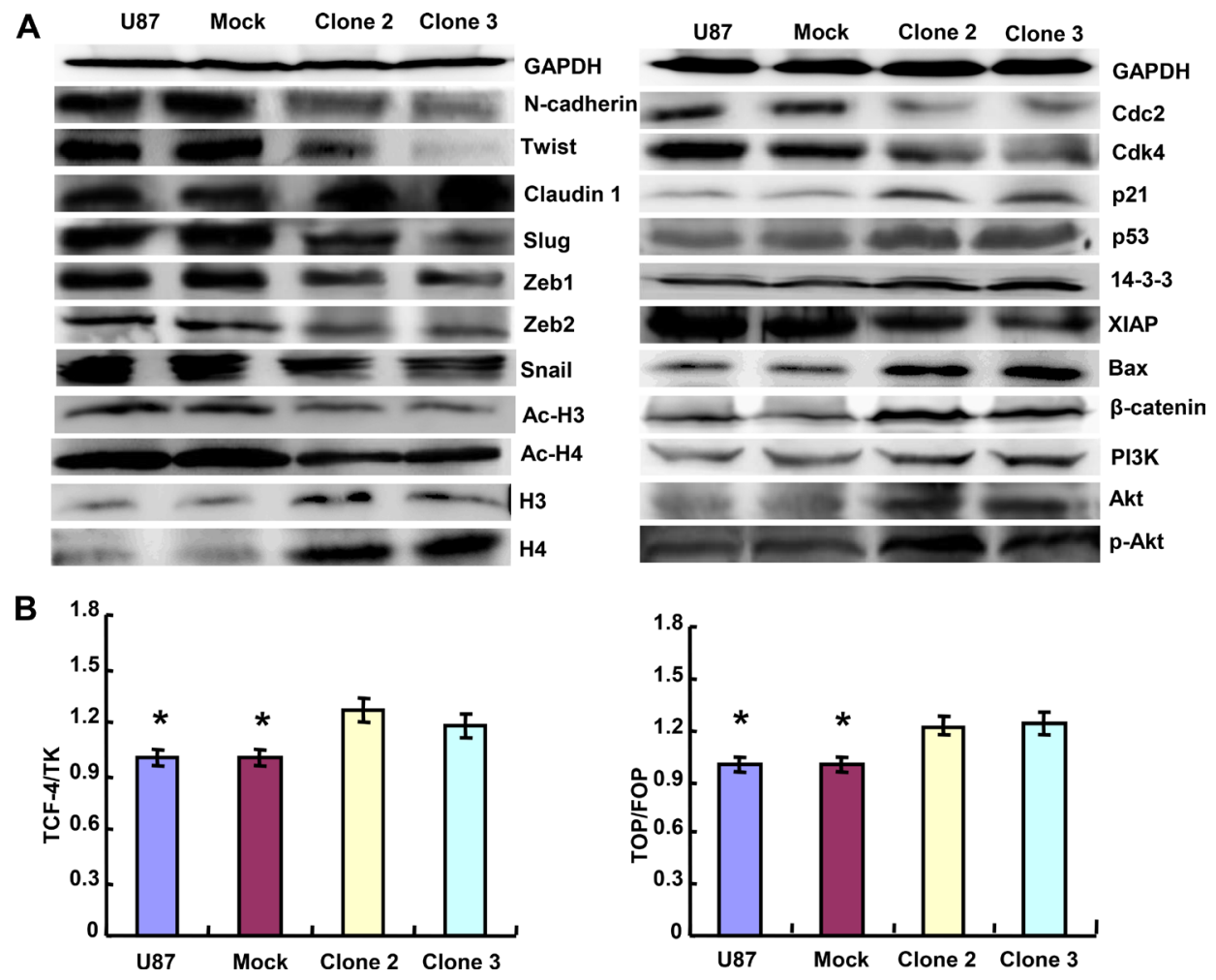

Figure 3: ING5 altered the expression and transcriptional activity of phenotypes-related proteins in U87 cells. The phenotype-related molecules were screebed by western blot analysis (A). Dual luciferase gene assay demonstrated that ING5 increased TCF-4 promoter activity and TCF-4-mediated gene transcription activity (B). ${ }^{*} p<0.05$. 
and TCF-4 promoter activity and TCF4-mediated gene transcription activity were increased in ING5 transfectants. Taken together, it was suggested that the activating effects of ING5 on both $\mathrm{PI}_{3} \mathrm{~K} / \mathrm{Akt}$ and $\beta$-catenin/TCF-4 signal pathways contributed to chemotherapeutic resistance in glioma cells.

Both Cyclin D and E activate CDKs and promoted $\mathrm{G}_{1}-\mathrm{S}$ transition, which is inhibited by $\mathrm{p} 21$ and $\mathrm{p} 27$. Cdc25B activates the cyclin dependent kinase Cdc2 for entry into mitosis $[10,13]$. p53 protein can arrest growth by holding the cell cycle at the $\mathrm{G}_{1} / \mathrm{S}$ regulation point and initiate and senescence [25]. Therefore, p21 and p53 overexpression, as well as $\mathrm{Cdk} 4$ and $\mathrm{Cdc} 2$ hypoexpression would be responsible for the effects of ING5 on the $\mathrm{G}_{2} / \mathrm{M}$ arrest. According to the literatures [26, 27], two truncated fragments of ING5 (aa 1-184 and aa 107-226) can induce cellular senescence and S arrest by down-regulating Cyclin E and Cdk2 expression, while microRNA-193 was found to have pro-proliferation effects for bone mesenchymal stem cells after low-level laser irradiation treatment by targeting ING5 to regulate Cdk2 activity. Bax opens the mitochondrial voltage-dependent anion channel for apoptosis, while XIAP might bind to and suppress Caspase activation [10, 13]. Our investigation indicated that higher Bax expression and lower XIAP expression enhanced apoptosis-inducing events of ING5 via mitochondrial pathway.

Three pathways have been reported to induce EMT, all of which function through three families of transcription factors, including Snail (Snail, Slug), Zeb (Zeb1, Zeb2) and basic Helix Loop Helix (E47, Twist and others) [28]. Claudin 1 is an integral membrane protein and a component of tight junction strands necessary for epithelial sheets [29]. Here, we found that ING5 up-regulated the Claudin 1 expression, but down-regulated the expression of N-cadherin, Slug, Snail, Twist, Zeb1 and Zeb2, indicating that ING5 suppressed the EMT in glioma. Zhao et al. [11] found that ING5 inhibited EMT of breast cancer via $\mathrm{PI}_{3} \mathrm{~K} / \mathrm{Akt}$ pathway, in agreement with a report about the role of ING5 in lung cancer. It might give a rational explanation for the inhibitory effects of ING5 on migration, invasion and metastasis of cancer cells.

In summary, ING5 overexpression might suppress the proliferation, energy metabolism, migration, invasion, EMT and tumor growth, and induce apoptosis and chemotherapeutic resistance of glioma cells. Downregulated ING5 expression might be closely linked the tumorigenesis of glioma, and its overexpression with the histogenesis of anaplastic astrocytoma. $\mathrm{PI}_{3} \mathrm{~K} / \mathrm{Akt}$ or $\beta$-catenin/TCF-4 activation might be positively linked to chemotherapeutic resistance, mediated by ING5.

\section{MATERIALS AND METHODS}

\section{Cell culture}

Glioma cells U87 were obtained from the ATCC (Manassas, VA, USA) and grown in a humidified atmosphere of $5 \% \mathrm{CO}_{2}$ at $37^{\circ} \mathrm{C}$ in MEM with $10 \%$ fetal bovine serum (FBS), $100 \mathrm{U} / \mathrm{ml}$ penicillin and $100 \mu \mathrm{g} /$ $\mathrm{ml}$. Cells were collected and subjected to total RNA and protein extraction. The cells were transfected with pEGFPN1-ING5, pEGFP-N1 vector or pEGFP-LC3B at $24 \mathrm{~h}$ after seeding on dishes, or selected by G418. To check the drug sensitivity, we exposed cells to SAHA, MG132, cisplatin and paclitaxel respectively.

\section{Proliferation assay}

U87 cells and clones were planted at $2.0 \times 10^{3}$ cells per well in 96-well plates and allowed to adhere. At the indicated time points, $20 \mu \mathrm{l}$ of $5 \mathrm{mg} / \mathrm{ml} \mathrm{MTT}$ was added to each well, the plates were incubated for $4 \mathrm{~h}$, and then measured at $490 \mathrm{~nm}$.

\section{Cell cycle analysis}

$1 \times 10^{6}$ cells were collected, washed by PBS twice and fixed in cold $1 \mathrm{~mL} \mathrm{75 \%}$ ethanol for at least $12 \mathrm{~h}$. Cells were washed twice with PBS again and incubated with $1 \mathrm{ml} \mathrm{RNase}(0.25 \mathrm{mg} / \mathrm{ml})$ for $1 \mathrm{~h}$ at $37^{\circ} \mathrm{C}$. Cells were resuspended in propidium iodide (PI) at a concentration of $50 \mu \mathrm{g} / \mathrm{ml}$ and cycle analysis was performed by flow cytometry.

\section{Apoptosis assay}

Flow cytometry was performed with PI and FITClabeled annexin V (KeyGEN Biotech, China) following the manufacturer's instructions. In brief, U87 and clone cells were cultured for $48 \mathrm{~h}$, resuspended in $200 \mu \mathrm{l} 1 \times$ Binding Buffer, incubated with $10 \mu \mathrm{l}$ FITC-Annexin V 15 min in the dark, and resuspended in $300 \mu 11 \times$ Bingding Buffer. After that, $5 \mu \mathrm{l}$ PI was added to each tube. Flow cytometry was performed within $1 \mathrm{~h}$ and apoptosis analyzed by FlowJo software.

\section{Alkaline phosphatase (ALP) activity}

ALP activity was used as a marker of differentiation. U87 and clone cells were harvested, broken and subjected to the determination of ALP activity and protein concentration using Diagnostics ALP reagent (Sigma, USA) and Biorad protein assay kit (USA). ALP activity was calculated as $U$ per $g$ of protein.

\section{Cellular energy metabolism}

Mitochondrial respiration and glycolysis were measured by Seahorse XF Extracellular Flux Analyzer. Respiration and glycolysis were respectively measured as the rate of oxygen consumption (OCR) and extracellular acidification (ECAR). According to the standard protocol, we injected drugs in the following order: $10 \mathrm{mM}$ glucose, 
$1.5 \mu \mathrm{M}$ oligomycin, $50 \mathrm{mM} 2-\mathrm{DG}$ for glycolysis assay and $1.5 \mu \mathrm{M}$ oligomycin, $2 \mu \mathrm{M}$ FCCP , $0.5 \mu \mathrm{M}$ Rotenone and antimycin A for mitochondrial respiration. The OCR and ECAR values were determined from 3 wells per sample.

\section{Wound healing assay}

Cell migration was assessed using a wound healing assay as a method described previously [13]. Briefly, cells were seeded at $1 \times 10^{6}$ per well in 6-well culture plates and incubated overnight. A scratch wound was made with a tip, and cultured in FBS-free media, photographed after $6 \mathrm{~h}$ and $12 \mathrm{~h}$. The scratch area was measured using image J software.

\section{Transwell chamber assay}

Comparative migration experiments were conducted using a matrigel-coated transwell inserts (BD Bioscience, USA). $2.5 \times 10^{5}$ cells were resuspended in $200 \mu \mathrm{l} \mathrm{serum-}$ free MEM and planted to each insert. $600 \mu \mathrm{l}$ of media was added to the lower chambers contained 10\% FBS. After incubation for $48 \mathrm{~h}$, the cells on the upper surface of the inserts were wiped away with a cotton swab. The cells on the lower surface of the membrane were washed twice with PBS, fixed with 4\% paraformaldehyde for $15 \mathrm{~min}$, washed with PBS again, and stained with crystal violet dye $(0.1 \%)$ for the measurement. For invasive assay, the procedures were the same as above excluding the matrigel coating insert (BD Bioscience).

\section{$\beta$-galactosidase staining}

Senescence was determined by senescence-associated $\beta$-galactosidase using a senescence cell histochemical staining kit (Beyotime, China). Briefly, U87 and clone cells were firstly fixed for $15 \mathrm{~min}$ in paraformaldehyde. After washing with PBS, cells were incubated with $\beta$-galactosidase staining solution at $37^{\circ} \mathrm{C}$ overnight, and then photographed after washing with PBS twice.

\section{Luciferase reporter assay}

Cells were seeded in 24-well dishes and luciferase reporter assay was performed after $48 \mathrm{~h}$ of transfection using Dual-Luciferase ${ }^{\circledR}$ Reporter Assay System (Promega,USA). The pGL3-TK was used as a negative control. TCF-4-mediated gene transcription activity was determined by the ratio of pGL3-OT to pGL3-OF
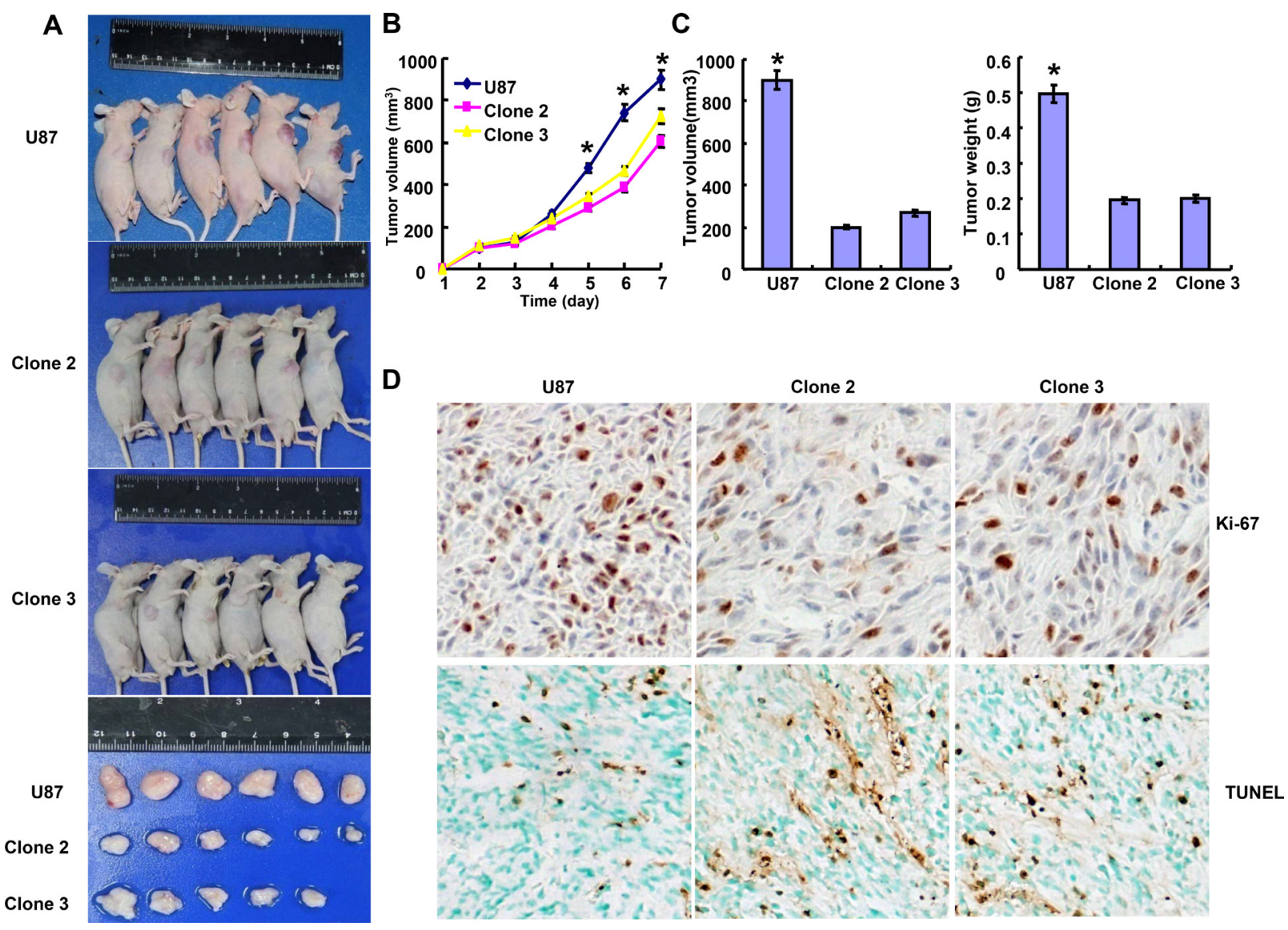

Figure 4: ING5 suppresses the tumor growth of glioma cells in vivo. The growth of U87 and clone cells were revealed by measurement of tumor size (A) and growth curve (B) volume and weight (C). Immunohistochemistry showed weaker Ki-67 staining and stronger TUNEL staining in clone cells (D). ${ }^{*} p<0.05$. 
luciferase activity, which was normalized to Renilla luciferase activity of the control plasmid, pRL-TK. TCF-4 promoter activity was determined by the value of $\mathrm{pGL}$ [1306] TCF4-Luc luciferase activity, which was also normalized by Renilla luciferase activity of pRL-TK.

\section{Real-time reverse transcriptase-polymerase chain reaction (real-time $\mathrm{RT}$-PCR)}

Total RNA was isolated from glioma cell lines using Trizol (Takara, Kyoto, Japan) and quantified in a Nanodrop spectrophotometer (Wilmington, USA). RT-PCR was performed from $2 \mu \mathrm{g}$ of total RNA using AMV reverse transcriptase and random primers (Takara). According to the Genbank (NM_032329.4), oligonucleotide primers for ING5 were designed as follows: forward: 5' GGGAGATGATTGGCTGTG-3' and reverse: 5'-CCTTTGGGTTTCGTGGTA-3' (614-759, 146bp). The primers for the internal control, GAPDH, were forward: 5'- CAATGAC CCCTTCATTGACC-3' and reverse: 5'-TGGAAGATGGTGATGGGATT-3' (201-335, $135 \mathrm{bp})$. Amplification of cDNA was performed using the SYBR Premix Ex Taq II kit (Takara) using GAPDH as an internal control.

\section{Western blot analysis}

Protein assays were performed by Kaumas brilliant blue method. The protein samples were resolved in $12 \%$ SDS-PAGE and electrotransferred to a PVDF membrane using standard procedures. The membrane was blocked with 5\% skimmed milk in Tris-buffered saline with Tween 20 (TBST) for $1 \mathrm{~h}$ and the primary antibody (Table 3 ) were added on the shaker at $4^{\circ} \mathrm{C}$ overnight. The membranes were rinsed with TBST, and incubated with anti-rabbit, anti-mouse or anti-goat IgG antibodies conjugated to horseradish peroxidase (HRP; Dako, USA) at a dilution of 1:5000 for $1 \mathrm{~h}$. Bands were visualized with LAS4010 (GE Life Science, USA) by ECL-Plus detection reagents (Santa Cruz, USA).

\section{A}
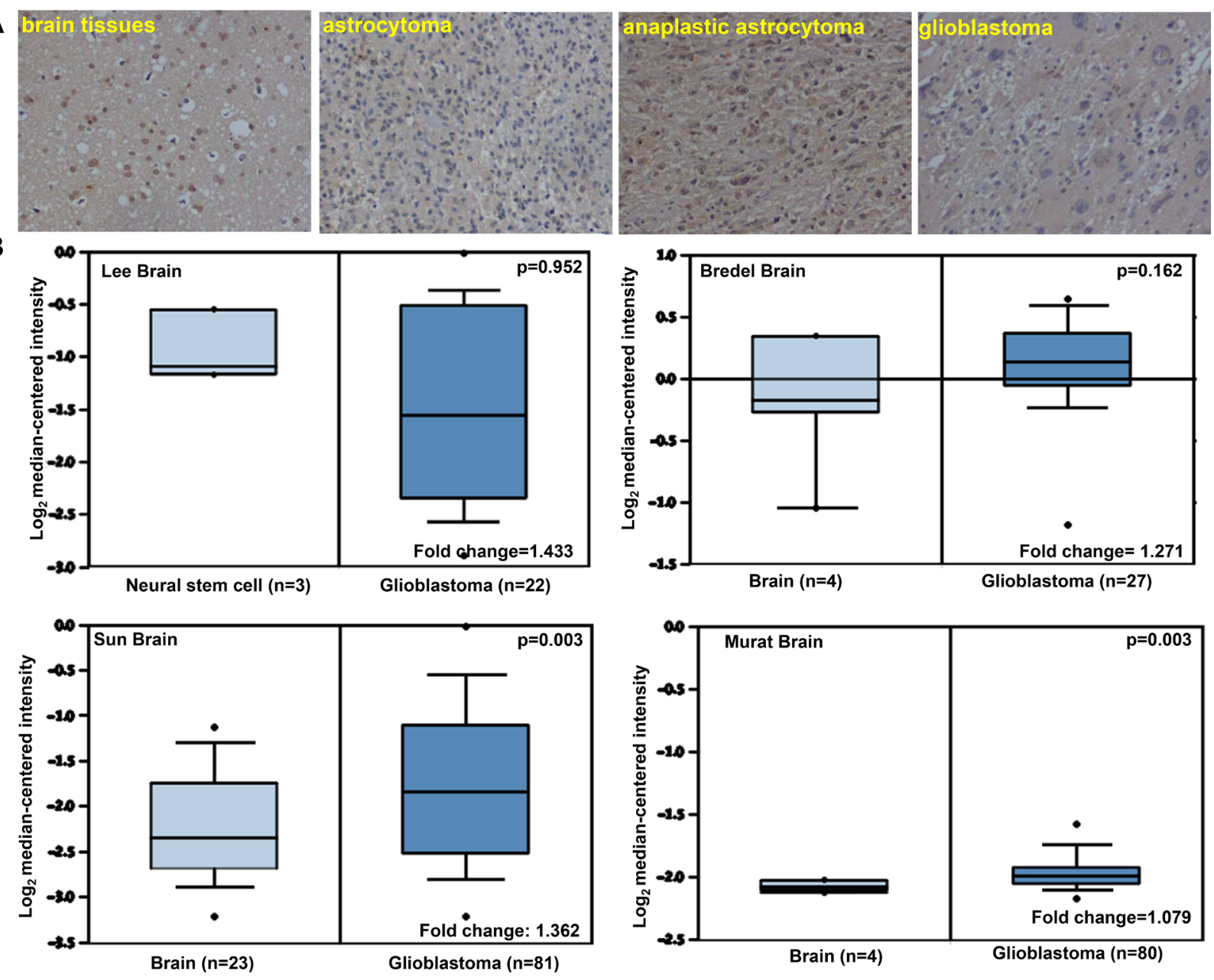

Figure 5: ING5 expression in gliomas. According to immunohistochemistry, the positivity to ING5 protein was detectable in the nucleus and cytoplasm of normal brain tissues, astrocytoma, anaplastic astrocytoma and glioblastoma (A). Lee's, Bredel's, Sun's and Murat's datasets were employed for bioinformatical analysis to compare ING5 mRNA expression between brain normal tissues and glioblastomas (B). 
Table 3: Antibodies used for western blot

\begin{tabular}{|c|c|c|}
\hline Name & Source & Company \\
\hline N-cadherin & Rabbit & Wanleibio \\
\hline Twist & Rabbit & Wanleibio \\
\hline Claudin 1（D-4） & Mouse & Santa Cruz Biotechnology \\
\hline Slug & Rabbit & Abcam \\
\hline Zeb1 & Rabbit & Wanleibio \\
\hline Zeb2（E-11） & Mouse & Santa Cruz Biotechnology \\
\hline Snail & Rabbit & Wanleibio \\
\hline Ac-histone 3 (Lys 9/14) & Goat & Santa Cruz Biotechnology \\
\hline Ac-histone 4 (Lys 8) & Rabbit & Santa Cruz Biotechnology \\
\hline Histone H3 (N-20) & Goat & Santa Cruz Biotechnology \\
\hline Histone H4 (H-97) & Rabbit & Santa Cruz Biotechnology \\
\hline Cdc2 (C-9) & Mouse & Santa Cruz Biotechnology \\
\hline Cdk4(C-22) & Rabbit & Santa Cruz Biotechnology \\
\hline p21 (F-5) & Mouse & Santa Cruz Biotechnology \\
\hline p53 & Rabbit & Wanleibio \\
\hline $14-3-3(\mathrm{H}-8)$ & Mouse & Santa Cruz Biotechnology \\
\hline XIAP (H-202) & Rabbit & Santa Cruz Biotechnology \\
\hline Bax (B-9) & Mouse & Santa Cruz Biotechnology \\
\hline$\beta$-catenin & Rabbit & Abcam \\
\hline PI3K & Rabbit & Abcam \\
\hline Akt1/2/3 (H-136) & Rabbit & Santa Cruz Biotechnology \\
\hline p-Akt1/2/3 (Thr 308) & Rabbit & Santa Cruz Biotechnology \\
\hline ING5 & Rabbit & Proteintech \\
\hline GAPDH & Rabbit & Wanleibio \\
\hline
\end{tabular}

\section{Selection of patient samples}

A total of 368 glioma cases were collected from surgical resection in The First Affiliated Hospital of Jinzhou Medical University $(n=108)$ between 2002 and 2014, and Shengjing Hospital of China Medical University, Shenyang, China $(n=260)$ between 2007 and 2014. The majority of samples were routinely prepared for storage in pathological blocks. None of the patients had undergone chemotherapy, radiotherapy or adjuvant treatment prior to surgery. Informed written consent was obtained from all participants and the study was approved by the Ethics Committees of both universities.

\section{Pathology and tissue microarray (TMA) analysis}

All tissues were fixed in $10 \%$ neutral formalin, embedded in paraffin and sliced into $4 \mu \mathrm{m}$-thick sections. The sections were stained with hematoxylin-and-eosin (HE) to for histological analysis. The pathological staging or histological classification was evaluated according to the World Health Organization classification system.

Representative areas of solid tumors were identified in the HE-stained slides of selected tumor samples. Tissue cores were punched out from each donor block and transferred to a recipient block using a Tissue
Microarrayer (AZUMAYA KIN-1, Tokyo, Japan). Each recipient block had a maximum of 70 cores. Consecutive $4 \mu \mathrm{m}$-thick sections were incised from the recipient block and transferred to poly-lysine-coated glass slides.

\section{Xenograft models}

Female Balb/c nude mice of 6-8 weeks were kept in a specific pathogen-free (SPF) facility with a $12 \mathrm{~h}$ light/ dark cycle. All animal treatments were in accordance with National Institutes of Health Care and Use of Laboratory Animals. This study was approved by the Institutional Animal Care and Use Committee of our hospital.

U87 and clone cells were grown, detached by trypsinization, followed by washing and re-suspended in serum-free MEM. Subcutaneous xenografts were established by injection of $1 \times 10^{6}$ cancer cells /mouse to the axilla ( $n=$ 10 /group). Tumor growth was then monitored for 7 days and calculated as follows: length $\times$ width $\times$ width $\times 0.5$. At the end of the experiment, mice from each group was anesthetized, photographed, and sacrificed for further analysis. The volume and weight of xenograft tumor were determined by capacity measurement and weighting. The tumor tissues were fixed in $10 \%$ neutral formalin and subjected to the routine block preparation for the following experiments. 


\section{Immunohistochemistry (IHC)}

IHC was carried out on $4 \mu$ m-thick sections of glioma TMAs were deparaffinized with xylene, rehydrated with alcohol, and subjected to antigen retrieval by heating in target retrieval solution (TRS, Dako) for $20 \mathrm{~min}$ in a microwave oven. The sections were quenched with $3 \%$ hydrogen peroxide for $5 \mathrm{~min}$ to block endogenous peroxidase activity. Non-specific binding was prevented by $5 \%$ bovine serum albumin for 5 min.

The sections were incubated with rabbit anti-ING5 (Proteintech, USA) or anti-ki-67 (Dako, USA) for $1 \mathrm{~h}$, and then incubated with anti-rabbit antibodies conjugated to HRP (Dako) for $1 \mathrm{~h}$. After each treatment all sections were washed three times with TBST and the binding sites were visualized with DAB. After counterstaining with Mayer's hematoxylin, the sections were dehydrated, cleared and mounted.

Three independent observers (ZS, ZZJ and ZHC) randomly selected five representative fields from each section. Any discrepancies were checked by both observers until a consensus was reached. Positive expression was graded as follows: $-=$ negative; $+=1 \%-50 \% ;++=51 \%-$ $74 \% ;+++\geq 75 \%$.

\section{TUNEL}

Terminal deoxynucleotide transferase mediated dUTP nick labeling (TUNEL) was performed using Apoptosis Detection Kit (Millipore, USA). Briefly, the sections were incubated with proteinase $\mathrm{K}$ at $37^{\circ} \mathrm{C}$ for $30 \mathrm{~min}$. Endogenous peroxidase activity was blocked by incubation with $3 \%$ hydrogen peroxide in methanol, and then subjected to TUNEL staining. The conjugated horseradish peroxidase was visualized with diaminobenzidine, followed by counterstaining with methyl green.

\section{Oncomine analysis}

The individual gene expression level of ING5 was analyzed using Oncomine (www.oncomine.org), which a cancer microarray database and web-based data mining platform for a new discovery from genome-wide expression analyses. We compared the differences in ING5 mRNA level between normal brain tissue and glioblastoma according the data provided by the authors upon request. All data were log-transformed, median centered per array, and standard deviation normalized to one per array.

\section{Statistical analysis}

Statistical evaluation was performed using Spearman's rank correlation to analyze rank data, and
Mann-Whitney $U$ to differentiate the means of different groups. A $p$-value $<0.05$ was considered statistically significant. SPSS v. 10.0 software was employed to analyze all data.

\section{ACKNOWLEDGMENTS AND FUNDING}

This study was supported by Liaoning BaiQianWan Talents Program, Award for Liaoning Distinguished Professor, A Key Scientific and Technological Project of Liaoning Province (2015408001) and National Natural Scientific Foundation of China (81472544; 81672700).

\section{CONFLICTS OF INTEREST}

The authors declare that they have no competing interests.

\section{REFERENCES}

1. Zhang Y, Zhao S, Xu Z. Network and pathway analysis of microRNAs, transcription factors, target genes and host genes in human glioma. Oncol Lett. 2016; 11:3534-3542.

2. Roy B, Gupta RK, Maudsley AA, Awasthi R, Sheriff S, Gu M, Husain N, Mohakud S, Behari S, Pandey CM, Rathore RK, Spielman DM, Alger JR. Utility of multiparametric 3-T MRI for glioma characterization. Neuroradiology. 2013; 55:603-613.

3. Cuddapah VA, Robel S, Watkins S, Sontheimer H. A neurocentric perspective on glioma invasion. Nat Rev Neurosci. 2014; 15:455-465.

4. Soliman MA, Riabowol K. After a decade of study-ING, a PHD for a versatile family of proteins. Trends Biochem Sci. 2007; 32:509-519.

5. Campos EI, Chin MY, Kuo WH, Li G. Biological functions of the ING family tumor suppressors. Cell Mol Life Sci. 2004; 61:2597-2613.

6. Russell M, Berardi P, Gong W, Riabowol K. GrowING, Age-ING and Die-ING: ING proteins link cancer, senescence and apoptosis. Exp Cell Res. 2006; 312:951-961.

7. Linzen U, Lilischkis R, Pandithage R, Schilling B, Ullius A, Lüscher-Firzlaff J, Kremmer E, Lüscher B, Vervoorts J. ING5 is phosphorylated by CDK2 and controls cell proliferation independently of p53. PLoS One. 2015; 10: e0123736.

8. Zhang F, Bäumer N, Rode M, Ji P, Zhang T, Berdel WE, Müller-Tidow C. The inhibitor of growth protein 5 (ING5) depends on INCA1 as a co-factor for its antiproliferative effects. PLoS One. 2011; 6:e21505.

9. Cengiz B, Gunduz E, Gunduz M, Beder LB, Tamamura R, Bagci C, Yamanaka N, Shimizu K, Nagatsuka H. Tumorspecific mutation and downregulation of ING5 detected in oral squamous cell carcinoma. Int J Cancer. 2010; 127: 2088-2094. 
10. Zhao S, Yang XF, Shen DF, Gao Y, Shi S, Wu JC, Liu HX, Sun HZ, Su RJ, Zheng HC. The down-regulated ING5 expression in lung cancer: a potential target of gene therapy. Oncotarget. 2016; 7:54596-54615. doi: 10.18632/ oncotarget. 10519.

11. Zhao QY, Ju F, Wang ZH, Ma XZ, Zhao H. ING5 inhibits epithelial-mesenchymal transition in breast cancer by suppressing PI3K/Akt pathway. Int J Clin Exp Med. 2015; 8:15498-15505.

12. Li Y, Deng H, Lv L, Zhang C, Qian L, Xiao J, Zhao W, Liu Q, Zhang D, Wang Y, Yan J, Zhang H, He Y, et al. The miR193a-3p-regulated ING5 gene activates the DNA damage response pathway and inhibits multi-chemoresistance in bladder cancer. Oncotarget. 2015; 6:10195-10206. doi: 10.18632/oncotarget.3555.

13. Gou WF, Shen DF, Yang XF, Zhao S, Liu YP, Sun HZ, Su RJ, Luo JS, Zheng HC. ING5 suppresses proliferation, apoptosis, migration and invasion, and induces autophagy and differentiation of gastric cancer cells: a good marker for carcinogenesis and subsequent progression. Oncotarget. 2015; 6:19552-19579. doi: 10.18632/oncotarget.3735.

14. Zhang F, Zhang X, Meng J, Zhao Y, Liu X, Liu Y, Wang Y, Li Y, Sun Y, Wang Z, Mei Q, Zhang T. ING5 inhibits cancer aggressiveness via preventing EMT and is a potential prognostic biomarker for lung cancer. Oncotarget. 2015; 6: 16239-16252. doi: 10.18632/oncotarget.3842.

15. Li X, Nishida T, Noguchi A, Zheng Y, Takahashi H, Yang X, Masuda S, Takano Y. Decreased nuclear expression and increased cytoplasmic expression of ING5 may be linked to tumorigenesis and progression in human head and neck squamous cell carcinoma. J Cancer Res Clin Oncol. 2010; 136:1573-1583.

16. Cao Y, Chen J, Wang D, Peng H, Tan X, Xiong D, Huang A, Tang H. Upregulated in Hepatitis B virus-associated hepatocellular carcinoma cells, miR-331-3p promotes proliferation of hepatocellular carcinoma cells by targeting ING5. Oncotarget. 2015; 6:38093-38106. doi: 10.18632/ oncotarget.5642.

17. Liu M, Du Y, Gao J, Liu J, Kong X, Gong Y, Li Z, Wu H, Chen H. Aberrant expression miR-196a is associated with abnormal apoptosis, invasion, and proliferation of pancreatic cancer cells. Pancreas. 2013; 42:1169-1181.

18. Zheng HC, Xia P, Xu XY, Takahashi H, Takano Y. The nuclear to cytoplasmic shift of ING5 protein during colorectal carcinogenesis with their distinct links to pathologic behaviors of carcinomas. Hum Pathol. 2011; 42:424-433.
19. Xing YN, Yang X, Xu XY, Zheng Y, Xu HM, Takano Y, Zheng HC. The altered mexpression of ING5 protein is involved in gastric carcinogenesis and subsequent progression. Hum Pathol. 2011; 42:25-35.

20. Shiseki M, Nagashima M, Pedeux RM, KitahamaShiseki M, Miura K, Okamura S,Onogi H, Higashimoto Y, Appella E, Yokota J, Harris CC. p29ING4 and p28ING5 bind to p53 and p300, and enhance p53 activity. Cancer Res. 2003; 63:2373-2378.

21. Mendes-Pereira AM, Sims D, Dexter T, Fenwick K, Assiotis I, Kozarewa I, Mitsopoulos C, Hakas J, Zvelebil M, Lord CJ, Ashworth A. Genome-wide functional screen identifies a compendium of genes affecting sensitivity to tamoxifen. Proc Natl Acad Sci USA. 2012; 109:2730-2735.

22. Helmreic EJM. The Biochemistry of Cell Signaling. Oxford: OUP; 2001. 200:242.

23. Hernandez-Aya LF, Gonzalez-Angulo AM. Targeting the phosphatidylinositol 3-kinase signaling pathway in breast cancer. Oncologist. 2011; 16:404-414.

24. Xu XY, Xia P, Yu M, Nie XC, Xing YN, Liu YP, Takano Y, Zheng HC. The roles of REIC gene and its encoding product in gastric carcinoma. Cell Cycle. 2012; 11:1414-1431.

25. Charni M, Aloni-Grinstein R, Molchadsky A, Rotter V. p53 on the crossroad between regeneration and cancer. Cell Death Differ. 2017; 24:8-14.

26. Qi L, Zhang Y. Truncation of inhibitor of growth family protein 5 effectively induces senescence, but not apoptosis in human tongue squamous cell carcinoma cell line. Tumour Biol. 2014; 35:3139-3144.

27. Wang J, Huang W, Wu Y, Hou J, Nie Y, Gu H, Li J, Hu S, Zhang H. MicroRNA-193 pro-proliferation effects for bone mesenchymal stem cells after low-level laser irradiation treatment through inhibitor of growth family, member 5 . Stem Cells Dev. 2012; 21:2508-2519.

28. Peinado H, Olmeda D, Cano A. Snail, Zeb and bHLH factors in tumour progression: an alliance against the epithelial phenotype? Nat Rev Cancer 2007; 7:415-428.

29. Zhou B, Moodie A, Blanchard AA, Leygue E, Myal Y. Claudin 1 in breast cancer: new insights. J Clin Med. 2015; 4:1960-1976. 